\title{
Hamilton's Principle for Circuits with Dissipative Elements
}

\author{
Zdeněk Biolek $\left(\mathbb{D},{ }^{1}\right.$ Dalibor Biolek $\left(\mathbb{D},{ }^{1,2}\right.$ and Viera Biolková $\mathbb{D}^{3}$ \\ ${ }^{1}$ Department of Microelectronics, Brno University of Technology, Brno 616 00, Czech Republic \\ ${ }^{2}$ Department of Electrical Engineering, University of Defence, Brno 662 10, Czech Republic \\ ${ }^{3}$ Department of Radioelectronics, Brno University of Technology, Brno 616 00, Czech Republic
}

Correspondence should be addressed to Dalibor Biolek; dalibor.biolek@unob.cz

Received 12 August 2019; Accepted 8 October 2019; Published 5 December 2019

Academic Editor: Christos Volos

Copyright (C) 2019 Zdeněk Biolek et al. This is an open access article distributed under the Creative Commons Attribution License, which permits unrestricted use, distribution, and reproduction in any medium, provided the original work is properly cited.

\begin{abstract}
The classic form of Hamilton's variational principle does not hold for circuits with dissipative elements. It is shown in the paper that this may not be true in the case of systems consisting of the so-called higher-order elements. Hamilton's principle is then extended to circuits containing the classical resistors and Frequency Dependent Negative Resistors (FDNRs). The extension is also made to any pair of elements which are the nearest neighbours on any $\Sigma$-diagonal of Chua's table.
\end{abstract}

\section{Introduction}

Hamilton's variational principle is one of the jewels of classical mechanics [1]. Let $L$ be a Lagrange function of the system (Lagrangian) dependent on the generalized coordinates $q_{i}$ and velocities $\mathrm{d} q_{i} / \mathrm{d} t, i=1, \ldots, n$, where $n$ is the number of degrees of freedom. The well-known form of the Lagrangian is the difference between the kinetic and the potential energy of the system. Total action is taken to mean the definite integral:

$$
A=\int_{t_{1}}^{t_{2}} L\left(q_{1}, \ldots, q_{n}, \dot{q}_{1}, \ldots, \dot{q}_{n}, t\right) \mathrm{d} t
$$

where $t_{1}$ and $t_{2}$ are the endpoints of the time interval over which the analysis is performed. The action (1) is a functional, which maps the function $L$ (trajectory-dependent Lagrangian) to the number $A$ (action value assigned to a particular trajectory). Hamilton's principle says that the real trajectory is an extremal of the action, i.e., the variations made around the real trajectory lead to the zero value of the variation of the action:

$$
\delta A=\delta \int_{t_{1}}^{t_{2}} L \mathrm{~d} t=0
$$

For a transition between two states, the system seems to be searching for a trajectory for which the action integral acquires a stationary value. For one degree of freedom, the situation is illustrated in Figure 1. The real trajectory $q(t)$ is "pinheld" at the points A and B, and other trajectories $q(t)+$ $\delta q(t)$ are created via arbitrary deformations in a vertical direction. According to (1), the action $A$ can be calculated for each of these trajectories. The existing trajectory is the one for which the action acquires a stationary value (minimum, maximum, or saddle point). For a system with $n$ degrees of freedom, the existing trajectory $\left(q_{1}(t), \ldots, q_{n}(t)\right)$ is therefore a solution to the optimization problem (2).

It is well-known that condition (2) is equivalent to another condition, namely, the equations of motion of the system are in the form of the Euler-Lagrange equations (E-L) [1]

$$
\frac{\mathrm{d}}{\mathrm{d} t}\left(\frac{\partial L}{\partial \dot{q}_{i}}\right)-\frac{\partial L}{\partial q_{i}}=0
$$

These equations of motion represent the equilibrium of the so-called generalized forces [1]. For the Lagrangian in the form of a difference between the kinetic and the potential energy, the first left-side term of equation (3) is the derivative of the momentum with respect to time, or the inertial force, and the second term represents the conservative force originating from the potential energy of the system. From just one scalar function $L$, formula (3) generates all the generalized forces and puts them into the equation of motion. 


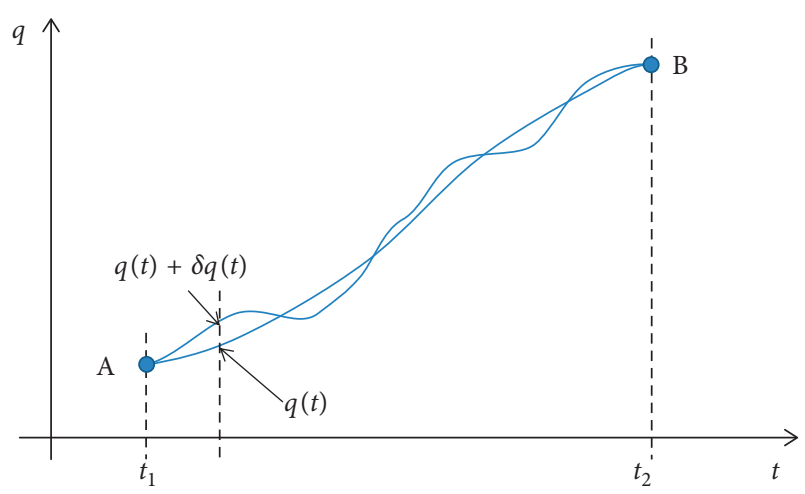

FIgURE 1: Virtual trajectory (thin line) differs from real trajectory (thick line) by the virtual deviation $\delta q(t)$, which is zero in the start and end states $\mathrm{A}$ and $\mathrm{B}$.

However, nonconservative forces cannot be generated from a Lagrangian that is dependent on coordinates and velocities [1]. A frequently quoted claim is therefore that the validity of Hamilton's principle (2) is limited to conservative systems [1]. Much effort has been devoted to finding such a Lagrangian that would also generate nonconservative forces. A summary of the results can be found in [2], which also offers a solution in the form of a Lagrangian dependent on the noninteger order of the derivative of a generalized coordinate. In this work, we will use the classical form of the Lagrangian, which is dependent on the generalized coordinates and velocities, the latter being the first and thus integer-order derivatives of the coordinates.

Hamilton's variational principle also holds for systems with external excitations. The potential energy and therefore the Lagrangian $L$ are completed by terms that, after their appropriate differentiation, generate the original excitation force in the equations of motion (3) [1]. Without loss of generality, only systems without external excitation will be therefore considered in the following text.

Lagrange's and Hamilton's formalisms represent an elegant approach to studying the system dynamics. That is why it is preferred when constructing modern physical theories. As the characteristic attribute of Lagrange's formalism, the entire information on the time evolution of a system is contained in the scalar function-the Lagrangian. If the Lagrangian exists, and if it can be found, then it can generate the equations of motion of the system. Hamilton's formalism can also provide information on the quantities that are conserved in the system, on important symmetries, and so forth. No wonder that "Today most physicists would be not only willing to accept as axiomatic the existence of a variational principle but would be also loath to accept any dynamical equations that were not derivable from such a principle" [3]. This paper aims to show that the benefits of Lagrange's formalism can be also used for dissipative systems compounded of classical resistors and the so-called FDNRs, the frequency dependent negative resistors, from Chua's periodical table of fundamental electrical elements [4]. The paper is organized as follows: Section 2 introduces a notation that is suitable for the utilization of Lagrange's formalism in electrical circuits containing not only reactive but also dissipative elements. Section 3 summarizes information on the table of fundamental electrical elements. In Section 4, Hamilton's principle is proved for circuits compounded of resistors and FDNRs, and the corresponding Lagrangian is found. The explanation that the duality principle enables a generalization of these conclusions also to circuits containing other elements from Chua's table than resistors and FDNRs is given in Section 5.

\section{Notation for Lagrange's Formalism in Electrical Engineering}

In addition to mechanics, Hamilton's principle is also used in other branches of science. In electric circuits described by methods of loop variables, the coordinate $q$ corresponds to the electric charge and the equation of motion (3) reflects the voltage balance, i.e., it represents Kirchhoff's Voltage Law (KVL). Within this electromechanical analogy, mechanical inertness corresponds to electrical inductance and mechanical compliance to electrical capacity. Conservative systems of mechanical nature can therefore be successfully studied and modelled using inductors and capacitors. If the electric circuit only consists of these elements, then the Lagrange function can be chosen as the difference $L=T^{*}-V$ between the coenergy of magnetic fields of inductors and the energy of the electrostatic fields of capacitors, which are integrals of the generally nonlinear constitutive relations $f_{L}$ and $f_{C}$ of inductors and capacitors:

$$
\begin{aligned}
T^{*} & =\sum_{L} \int f_{L}\left(\dot{q}_{L}\right) \mathrm{d} \dot{q}_{L}, \\
V & =\sum_{C} \int f_{C}\left(q_{C}\right) \mathrm{d} q_{C} .
\end{aligned}
$$

The following notation is used in (4): the indices $L$ and $C$ used in the variables and functions and also as counting indices of the sums denote the constitutive relations of individual inductors and capacitors in the network. The charge/current through each individual element is given by a linear combination of the components of the vector of loop charges/currents:

$$
\begin{aligned}
& q_{C}=\sum_{i=1}^{n} a_{i} q_{i}, \\
& \dot{q}_{L}=\sum_{i=1}^{n} b_{i} \dot{q}_{i} .
\end{aligned}
$$

The coefficients $a_{i}, b_{i}$ take the values $+1,-1$, and 0 , depending on the incidence of the element in the corresponding loop and the reference direction of the element with regard to the loop orientation.

The dissipative resistive elements are characterized by the constitutive relations $f_{R}$ between voltages and currents. Their integrals are dissipative functions of currents, and their sum gives the total dissipative function:

$$
{ }^{+} D=\sum_{R} \int f_{R}\left(\dot{q}_{R}\right) \mathrm{d} \dot{q}_{R} .
$$


The subscript $R$ has the same meaning as the above indices $L$ and $C$. Currents flowing through individual resistors are also derived from the vector of loop currents by a linear combination of its components:

$$
\dot{q}_{R}=\sum_{i=1}^{n} c_{i} \dot{q}_{i}
$$

The coefficients $c_{i}$ take the values of $+1,-1$, and 0 , depending on whether the resistor is present/absent in the corresponding loop and on how its reference is directed with regard to the loop orientation. Additional terms are generated in the E-L equations (3) via a systematic differentiation of the dissipative function (6) with respect to the vector of currents. This, however, violates the necessary condition for the validity of Hamilton's variational principle.

\section{Higher-Order Elements}

The so-called $(\alpha, \beta)$ Higher-Order Elements (HOEs) were introduced in circuit theory in the 1980s [4]. The HOEs are one-ports, which preserve an unambiguous relation between the time derivatives/integrals of the terminal voltage and current $v^{(\alpha)}$ and $i^{(\beta)}$. The positive/negative integers $\alpha$ and $\beta$ stand for the order of the derivative/integral with respect to time. The properties and the character of the element are unambiguously given by the so-called constitutive relation as a generally nonlinear dependence relation between the $v^{(\alpha)}$ and $i^{(\beta)}$. Three fundamental elements of electrical engineering, namely, the resistor, capacitor, and inductor, are located in the diagram in Figure 2 in the $(0,0),(0,-1)$, and $(-1,0)$ coordinates. Figure 2 shows a fragment of the socalled Chua's table with presently known fundamental elements.

The memristor as the $(-1,-1)$ element appeared in 1971 [5]. In 2008, the discovery of a nanodevice that exhibits the signs of the behaviour of the memristor [6] initiated the process of filling the vacancies in the table with hitherto unknown elements. That same year, during a historical seminar [7], Leon Chua, the discoverer of the memristor, appealed to the scientific community to work on discovering new elements of the type of $(-1,-2)$ and $(-2,-1)$, which he termed memcapacitor and meminductor.

Another element termed FDNR (Frequency Dependent Negative Resistor) has been known since 1968 [8]. Its synthetic form is frequently used in active inductorless analog filters. Later on, this element was identified as the missing $(1,-1)$ element from Chua's table. Note that the FDNR from [8] is known as a linear element whose impedance is negative and indirectly proportional to square of the frequency, and the $(1,-1)$ element is its nonlinear generalization.

In 2002, the discovery of a new mechanical element termed inerter was announced in [9]. The scientists familiar with Chua's concept of fundamental electrical elements understood that it was the other missing element, either (1, 0 ) or $(0,1)$, depending on whether the F-V (force-voltage) or F-I (force-current) electromechanical analogy was used [10].

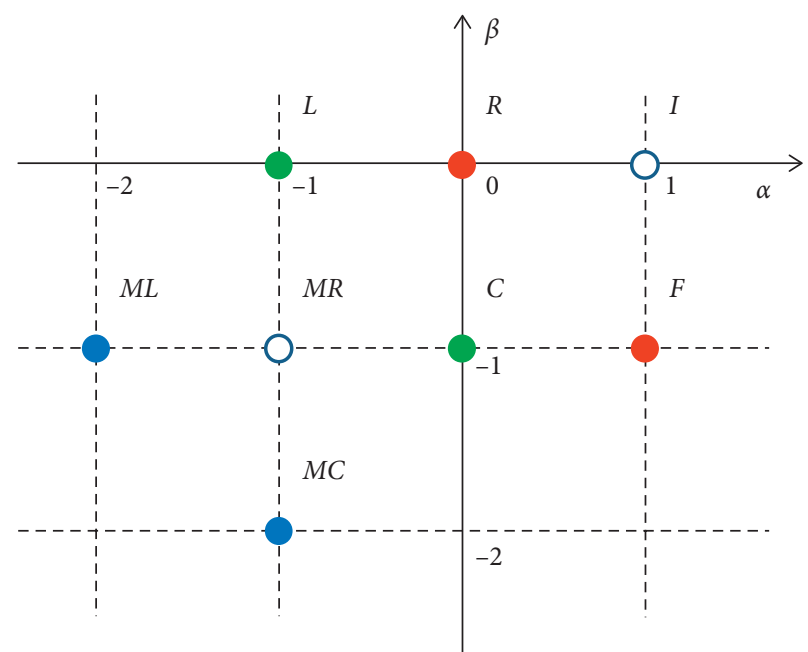

Figure 2: All currently known elements from Chua's table. $R, L$, $C=$ resistor, inductor, capacitor; $M R, M L, M C=$ memristor, meminductor, memcapacitor; $I, F=$ inerter, FDNR.

New mechanical and electrical elements bring a new inspiration to these disciplines. This paper demonstrates, on the example of a circuit consisting solely of resistors and FDNRs, that the classical version of Hamilton's principle can also be applied to some systems that contain dissipative elements.

\section{Hamilton's Principle for $R$-FDNR Circuits}

Consider a circuit consisting of ideal, generally nonlinear resistors, and Frequency Dependent Negative Resistors (FDNRs). The following consideration assumes the circuit to be described by the method of looped charges. However, the choice of the method is not essential.

Let each resistor be described by its constitutive relation $v=f_{R}(i)$, where the current $i$ is the time derivative of the charge $q$. Let the resistors in the circuit be described by a single dissipative function (6).

Furthermore, let each FDNR be defined by its constitutive relation $v^{(1)}=f_{F}\left(i^{(-1)}\right)$. According to the definition of the FDNR as a $(1,-1)$ element, its constitutive relation must be a relation between the first derivative of the terminal voltage and the total amount of the charge flowing through it. This unambiguous dependence makes it an element with a small-signal negative resistance, which is inversely proportional to the square of the excitation frequency.

According to the definition in [11], the "negative" dissipative cofunction of the whole circuit ${ }^{-} D^{*}$ is the sum of "negative" dissipative cofunctions of particular FDNRs:

$$
{ }^{-} D^{*}=\sum_{F} \int f_{F}\left(q_{F}\right) \mathrm{d} q_{F} .
$$

The charges $q_{F}$ associated with concrete elements can be derived from the vector of loop charges via linear combinations of its components: 


$$
q_{F}=\sum_{i=1}^{n} d_{i} q_{i}
$$

The coefficients $d_{i}$ take the values $+1,-1$, and 0 depending on which loop the individual FDNR is placed in and on its reference direction.

Now consider the vector variation of the trajectory $\left(\delta q_{1}, \ldots, \delta q_{n}\right)$ whose one-dimensional example is shown in Figure 1 . This variation concurrently means the variation of the constitutive relations of all FDNR's and thus also the variation of the dissipative cofunction (8):

$$
\delta\left(^{-} D^{*}\right)=\sum_{F} \sum_{i=1}^{n} \frac{\partial}{\partial q_{i}}\left(\int f_{F}\left(q_{F}\right) \mathrm{d} q_{F}\right) \delta q_{i} .
$$
form

The partial derivatives in (10) can be arranged in the

$$
\frac{\partial}{\partial q_{i}}\left(\int f_{F}\left(q_{F}\right) d q_{F}\right)=\frac{\mathrm{d}}{\mathrm{d} q_{F}}\left(\int f_{F}\left(q_{F}\right) \mathrm{d} q_{F}\right) \frac{\partial q_{F}}{\partial q_{i}} .
$$

The first factor (multiplicand) on the right side of (11) is the constitutive relation $f_{F}$. With equation (9) taken into account, the second factor (multiplier) is the coefficient $d_{i}$. After rearranging the order of summations, the variation of the cofunction (8) is equal to

$$
\delta\left(^{-} D^{*}\right)=\sum_{i=1}^{n}\left(\sum_{F} d_{i} f_{F}\left(q_{F}\right)\right) \delta q_{i}
$$

The inner sum in (12) gives the total contribution of the FDNR elements to $\mathrm{KV}^{(1)} \mathrm{L}$ law along the $i$-th loop.

A similar consideration of the effect of the trajectory variation on the dissipation function (6) leads to the intermediate result:

$$
\delta\left({ }^{+} D\right)=\sum_{i=1}^{n}\left(\sum_{R} c_{i} f_{R}\left(\dot{q}_{R}\right)\right) \delta \dot{q}_{i}
$$

The inner sum in (13) gives the total contribution of resistors to $\mathrm{KV}^{(0)} \mathrm{L}$ law along the $i$-th loop. The variation of the velocity takes place in (13) instead of the variation of coordinates. This imperfection can be corrected by integrating with respect to time, the same as Hamilton originally did for the kinetic energy in mechanics [1]. Via integrating by parts, integration with respect to velocity is changed to integration with respect to coordinate:

$$
\begin{aligned}
\delta \int_{t_{1}}^{t_{2}}\left({ }^{+} D\right) \mathrm{d} t= & {\left[\sum_{i=1}^{n}\left(\sum_{R} d_{i} f_{R}\left(\dot{q}_{R}\right)\right) \delta q_{i}\right]_{t_{1}}^{t_{2}} } \\
& -\int_{t_{1}}^{t_{2}} \sum_{i=1}^{n}\left(\frac{\mathrm{d}}{\mathrm{d} t} \sum_{R} d_{i} f_{R}\left(\dot{q}_{R}\right)\right) \delta q_{i} \mathrm{~d} t .
\end{aligned}
$$

Since this is a variational problem with fixed endpoints (the coordinates $q_{i}$ do not change at instants $t_{1}$ and $t_{2}$ ), the first element on the right side of (14) is zero. Integrating (12) with respect to time and subtracting from (14) yields

$$
\begin{aligned}
-\delta \int_{t_{1}}^{t_{2}}\left({ }^{+} D-{ }^{-} D^{*}\right) \mathrm{d} t= & \int_{t_{1}}^{t_{2}} \sum_{i=1}^{n}\left(\frac{\mathrm{d}}{\mathrm{d} t} \sum_{R} c_{i} f_{R}\left(\dot{q}_{R}\right)\right. \\
& \left.+\sum_{F} d_{i} f_{F}\left(q_{R}\right)\right) \delta q_{i} \mathrm{~d} t .
\end{aligned}
$$

The $i$-th term of the outer sum in (15) represents the sum of the first-order derivatives of voltages across all the elements within the $i$-th loop. With $\mathrm{KV}^{(1)} \mathrm{L}$ taken into consideration for this loop, this term must be equal to zero. The entire expression (15) must therefore be zero, irrespective of how the entire trajectory varies. Let us define the dissipative function $D$ of the whole circuit as the difference of the "positive" dissipative function of resistors and the "negative" dissipative cofunction of FDNRs:

$$
D={ }^{+} D-{ }^{-} D^{*} \text {. }
$$

Then, in a circuit built exclusively from resistors and FDNRs, the following variational principle holds:

$$
\delta \int_{t_{1}}^{t_{2}} D \mathrm{~d} t=0
$$

\section{Hamilton's Principle and Duality of $(\alpha, \beta)$ Elements}

Hamilton's variational principle (2) of classical mechanics has been formulated for generalized coordinates $q_{i}$ and generalized velocities $\mathrm{d} q i / \mathrm{d} t$. The E-L equation (3), which expresses the balance of generalized forces, is its direct consequence. Such generality made it useful also for other branches of science. The generalized coordinates and generalized forces can be chosen, with regard to the physical situation, very flexible. In electrical engineering, for example, the generalized coordinates/forces need not necessarily be charges $q /$ voltages $v$. The choice of a different method of circuit analysis, e.g., the method of node variables, leads to different coordinates and different generalized forces such as integrals of voltages (fluxes) and electric currents in the classical case of $R-C-L$ circuits. However, the situation changes if the $(\alpha, \beta)$ elements come onstage.

It is apparent from Figure 3 that a circuit made up exclusively of $L$ and $C$ elements (green color) will have its dual circuit made up exclusively of $R$ and FDNR elements (red color) and vice versa. If the functional rules of constitutive relations of each inductor and its corresponding resistor are the same for capacitors and FDNRs, then the equations of motion of both circuits will differ only in the index $\alpha$. In this case, equations describing an $L-C$ circuit will become equations of $R$-FDNR just by changing the voltage $v^{(0)}$ to its derivative, $v^{(1)}$. The transition from the $L-C$ to the dual $R$-FDNR circuit by increasing $\alpha$ is a special case of transformation, described in [12] as the MOVE transformation. Via this transformation, the original circuit can be modified such that the topology remains unchanged but each element is replaced by another element which is shifted in Chua's table by an offset $(\Delta \alpha, \Delta \beta)$, where $\Delta \alpha$ and $\Delta \beta$ can be 


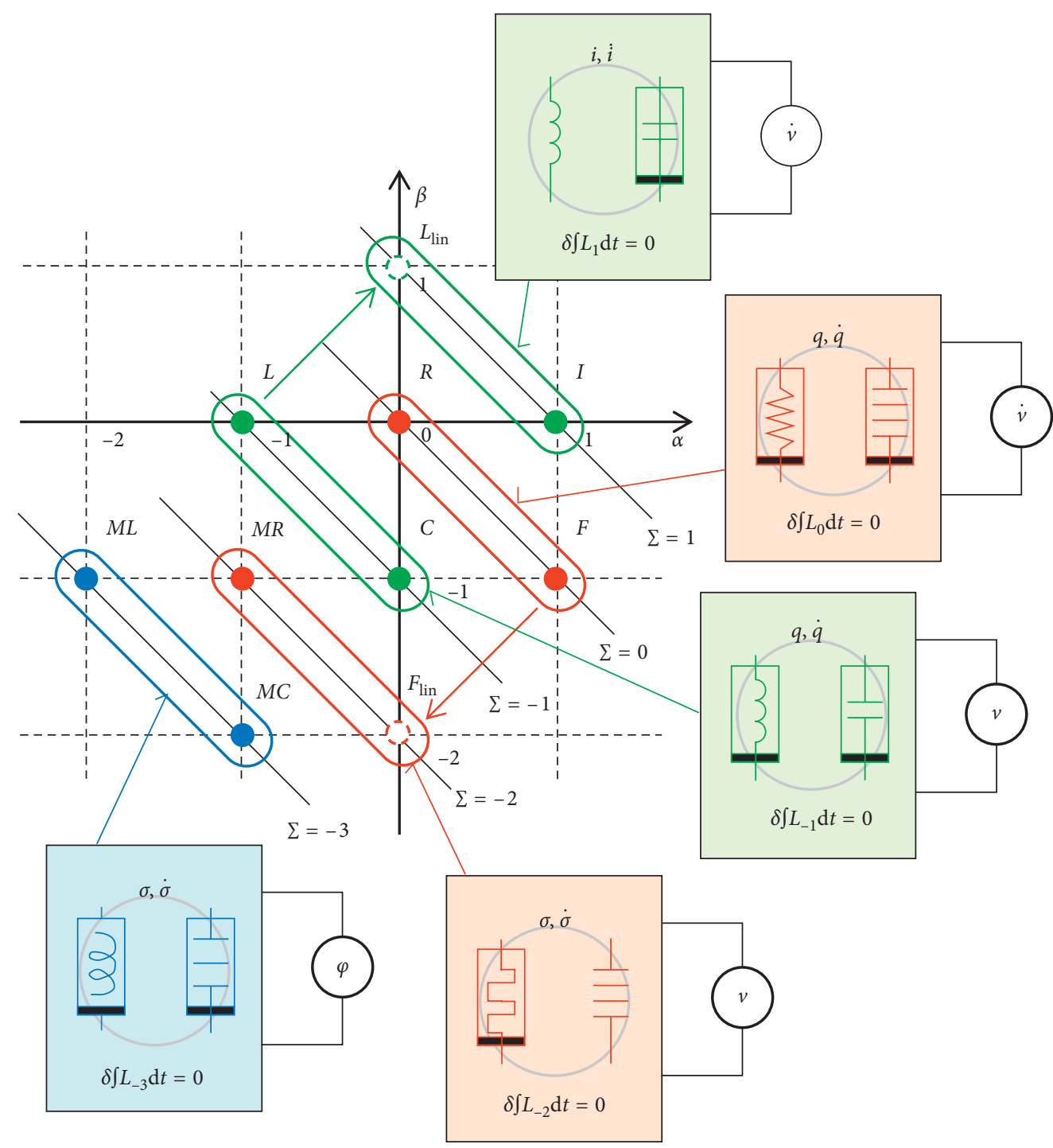

Figure 3: Circuits composed of color-marked pairs of elements located on $\Sigma=\alpha+\beta$ diagonals are mutually dual and Hamilton's variational principle applies to them. The symbol $L_{\Sigma}$ denotes the Lagrangian of the corresponding circuit. Note that $L_{0}$ is denoted in the text as a dissipative function $D$ of $R$-FDNR circuits.

arbitrary integers. The duality of such a transformation consists in the fact that when utilizing new generalized coordinates and velocities, the new circuit is governed by formally the same equations as the original circuit.

The MOVE transformation only changes the generalized coordinates, and it cannot have any effect on the validity of Hamilton's variational principle. This change will modify only the physical dimension of the Lagrange function, while its form remains unchanged. The color-coded pairs of the elements in Figure 3 are located on the common diagonals of the table, on which the sum $\Sigma=\alpha+\beta$ remains constant. One can easily check that the physical dimension of the Lagrangian of a circuit consisting of elements from the $\Sigma$-diagonal is equal to [VAs ${ }^{-\Sigma}$ ]. In $L-C$ circuits, $\Sigma=-1$ and the Langrangian has the dimension of energy $[\mathrm{VAs}]=[\mathrm{J}]$. Since $\Sigma=0$ in $R$-FDNR circuits, the role of the Lagrangian is superseded by the dissipative function with the physical dimension of power [VA].
As can be seen in Figure 3, dual $L-C$ and $R$-FDNR circuits share the same generalized coordinates and velocities, i.e., the loop charges and currents. The difference is in the physical dimensions of the terms of the loop equations of $\mathrm{KV}^{(0)} \mathrm{L}$ in the first and $\mathrm{KV}^{(1)} \mathrm{L}$ in the second case. This corresponds with the types of the prospective excitations via $v$ or $\mathrm{d} v / \mathrm{d} t$ sources.

The MOVE transformation can also yield a circuit made up entirely of $M L-M C$ elements (blue color in Figure 3). Hamilton's principle must hold for such a circuit as well. The $M L$ and $M C$ memory elements lie on the diagonal with $\Sigma=-3$, and the physical dimension of the Lagrangian $L_{-3}$ is $\left[\mathrm{Js}^{2}\right]$.

The $M L-M C$ circuit (blue color in Figure 3) can be obtained from an $L-C$ circuit by dual MOVE transformation, which decreases both the indices $\alpha$ and $\beta$ by 1 . The transition in $\alpha$-direction takes effect in changing the generalized coordinates from the original loop charges $q$ to their time 


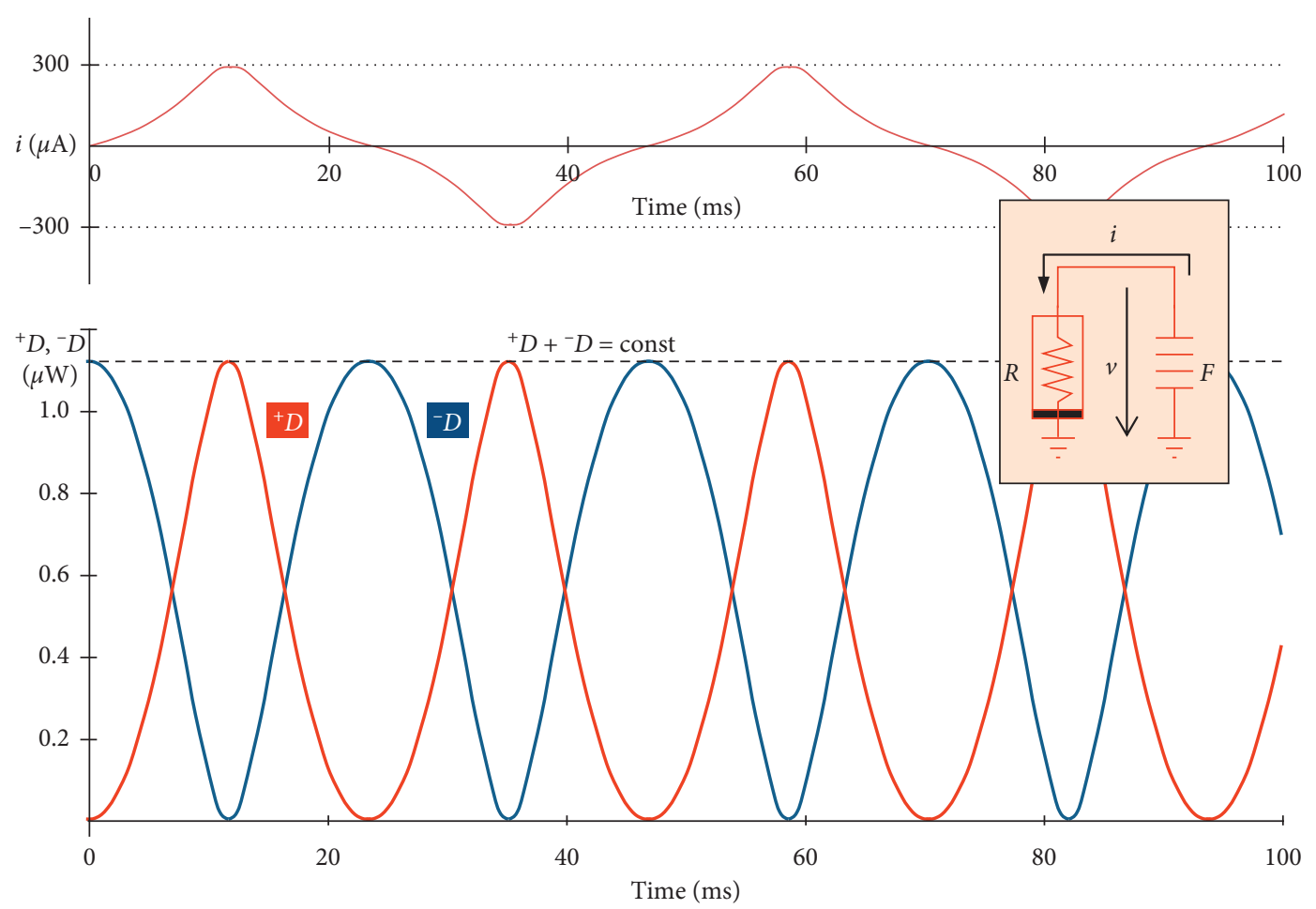

FIGURE 4: Analysis of the oscillator formed from a nonlinear resistor and a linear FDNR with the constitutive relations (18), $a=10 \mathrm{~m} \Omega$, $b=100 \mu \mathrm{A}$, and $c=1 \mathrm{M} \Omega \mathrm{s}^{-2}$. The waveforms of current (at the top) and dissipative function ${ }^{+} D$ of nonlinear resistor (6) and ${ }^{-} D$ of FDNR, the latter being equal (due to element linearity) to the dissipative cofunction ${ }^{-} D^{*}(8)$. The sum ${ }^{+} D+^{-} D$ is constant. It is in accordance with the content conservation law [14].

integrals $\sigma=q^{(-1)}$. The transition in $\beta$-direction will change the loop law from $\mathrm{KV}^{(0)} \mathrm{L}$ to $\mathrm{KV}^{(-1)} \mathrm{L}$, i.e., from voltage to flux balance. This corresponds with the types of prospective excitation in the form of flux sources $\varphi$. The same type of equations and the same link to analogous type of the Lagrangian imply the validity of Hamilton's principle, the same as for the classical case of $L-C$ circuits.

Examples of two other dual pairs, $M R-F_{\operatorname{lin}}$ and $L_{\operatorname{lin}}-I$, are shown in Figure 3. It is known that the elements located on the diagonal defined in Chua's table for constant difference $\alpha-\beta$ of indices share the same linearized model and that these elements are interconnected via the so-called SHIFT dual transformation [13]. That is why the $(0,-2)$ and $(0,1)$ elements in Figure 3 can be considered as linear FDNR and linear inductor. It is then obvious that two other pairs, namely, the memristor-linear FDNR and the linear inductorinerter, are also in accordance with Hamilton's principle.

Figure 4 illustrates the current and power conditions of the oscillator built from a nonlinear resistor and a linear FDNR in parallel. The nonlinear constitutive relation $v=$ $f_{R}(i)$ of the resistor and the linear constitutive relation $v^{(1)}=f_{F}(q)$ of the FDNR are in the forms

$$
\begin{aligned}
& f_{R}(i)=a \cdot \arctan \left(\frac{i}{b}\right), \\
& f_{F}(q)=c q,
\end{aligned}
$$

where $a, b$, and $c$ are parameters with values given in the legend of Figure 4.
The simulation results are shown in Figure 4. The oscillation with a period of $47 \mathrm{~ms}$ is induced by the initial condition $v^{(1)}(0)=1.5 \mathrm{Vs}^{-1}$. The oscillator fulfills the conditions of the validity of Hamilton's principle, and its Lagrangian is of $L_{0}$ type (see Figure 3 ). Even though the circuit is compounded exclusively of dissipative elements, it is conservative in the sense that it conserves the power quantity called the content [14], which in this case is equal to the sum of dissipative functions of the resistor and the FDNR. The dual circuit with the Lagrangian $L_{-1}$ is a classical LC oscillator, which conserves energy.

\section{Conclusion}

Within the classical mechanics, Hamilton's variational principle cannot be applied to dissipative processes. The same holds in the related scientific disciplines that have taken up this principle thanks to the fact that their specific generalized forces and coordinates are compatible with Hamilton's principle. Electrical engineering is no exception. Its link to mechanics through fundamental $R-C-L$ elements is well known and frequently used via electromechanical analogies.

In terms of Chua's table, Hamilton's principle is only given for systems consisting of $(0,-1)$ and $(-1,0)$ elements, which lie on the diagonal $\Sigma=-1$. It is well known that adding the dissipative element $(0,0)$, which lies on the diagonal $\Sigma=0$, into the above systems, cancels the validity of Hamilton's variational principle. 
Chua's table provides a new view on the fundamental electrical elements. It brings new challenges not only for electrical engineering, but also for mechanics. A new mechanical element, the inerter, discovered in 2002, cannot be substituted in its nonlinear form by any combination of known inertial, accumulative, or dissipative mechanical elements. While the classical inertia provides the algebraic bond between momentum and velocity, the inerter provides the algebraic bond between the derivatives of those quantities, i.e., between the acting force and acceleration. The classical mechanics did not take into account the existence of this fundamental law. The inerter is therefore a heterogeneous element for Hamilton's variational principle. As an element that lies on the diagonal $\Sigma=1$, it does not come up to the expectations that accompanied the birth of Hamilton's variational principle. To the best of our knowledge, no paper has been published that would formulate a variational principle involving this element.

It follows from the analysis in part IV that each circuit consisting of $C(0,-1)$ and $L(-1,0)$ elements is accompanied by its dual circuit consisting of the $(\alpha, \beta)$ and $(\alpha-1, \beta+1)$ elements, with $\alpha$ and $\beta$ being arbitrarily chosen indices. Note that the $(\alpha, \beta)$ and $(\alpha-1, \beta+1)$ elements lie on the common diagonal $\Sigma=\alpha+\beta$. Hamilton's variational principle must hold for all those dual circuits. The generalized forces and coordinates will be $v^{(\alpha)}$ and $i^{(\beta)}$, respectively.

Within the classical mechanics and electrical engineering, no other element exists for making a pair with the resistor-type dissipative element, which is necessary for the synthesis of systems that do not violate Hamilton's variational principle. It is shown in the paper that this missing element is the FDNR $(1,-1)$ element. Its linear form, the $(0$, $-2)$ element, creates a similar pair with the memristor. The applicability of Hamilton's variational principle to dissipative systems can therefore be considered only after the original set of the three fundamental $R, C, L$ elements has been extended by other elements from Chua's table.

Note that the validity of Hamilton's variational principle for systems consisting of any pair of elements which are the nearest neighbours on arbitrary $\Sigma$-diagonal of Chua's table is in accordance with the idea of generalized coordinates in classical mechanics. According to Noether's theorem [15], the quantity called generalized energy is conserved in such circuits. The generalized energy, having the physical unit $\left[\right.$ Volt $\cdot$ Amper $\left.\cdot \sec ^{-\Sigma}\right]$, is the sum of the potential functions of the elements of both types from the pairs in Figure 3. In the light of the above theorem, the conclusions made in the paper are in accordance with the rule that classical Hamilton's principle holds only for conservative systems.

\section{Data Availability}

No data were used to support this study.

\section{Acknowledgments}

This work was supported by the Czech Science Foundation under grant no. 18-21608S. For research, the infrastructure of K217 Department, UD Brno, was also used. The authors also wish to express their sincere thanks to the Open Access Fund of Brno University of Technology for covering the APC.

\section{References}

[1] C. Lanczos, The Variational Principles of Mechanics, Dover Books on Physics (Book 4), Dover Publications, New York, NY, USA, 4th Revised edition, 1986.

[2] F. Riewe, "Nonconservative Lagrangian and Hamiltonian mechanics," Physical Review E, vol. 53, no. 2, pp. 1890-1899, 1996.

[3] J. L. Anderson, Principles of Relativity Physics, Academic Press, New York, NY, USA, 1967.

[4] L. Chua, "Device modeling via nonlinear circuit elements," IEEE Transactions on Circuits and Systems, vol. 27, no. 11, pp. 1014-1044, 1980.

[5] L. Chua, "Memristor-the missing circuit element," IEEE Transactions on Circuit Theory, vol. 18, no. 5, pp. 507-519, 1971.

[6] D. B. Strukov, G. S. Snider, D. R. Stewart, and R. S. Williams, "The missing memristor found," Nature, vol. 453, no. 7191, pp. 80-83, 2008.

[7] L. O. Chua, "Memristors," in Proceedings of the 2nd Memristor and Memristive Systems Symposium, University of California, Berkeley, CA, USA, November 2008.

[8] L. T. Bruton, "Frequency selectivity using positive impedance converter-type networks," Proceedings of the IEEE, vol. 56, no. 8, pp. 1378-1379, 1968.

[9] M. C. Smith, "Synthesis of mechanical networks: the inerter," IEEE Transactions on Automatic Control, vol. 47, no. 10, pp. 1648-1662, 2002.

[10] D. Biolek, Z. Biolek, V. Biolková, and Z. Kolka, "Nonlinear inerter in the light of Chua's table of higher-order electrical elements," in Proceedings of IEEE Asia Pacific Conference on Circuits and Systems (APCCAS), pp. 617-620, Jeju, South Korea, October 2016.

[11] Z. Biolek, D. Biolek, and V. Biolková, "Euler-Lagrange equations of networks with higher-order elements," Radioengineering, vol. 26, no. 2, pp. 397-405, 2017.

[12] Z. Biolek, D. Biolek, V. Biolková, and Z. Kolka, "Taxicab geometry in table of higher-order elements," Nonlinear Dynamics, vol. 98, no. 1, pp. 623-636, 2019.

[13] D. Biolek, Z. Biolek, and V. Biolková, "Duality of complex systems built from higher-order elements," Complexity, vol. 2018, Article ID 5719397, 15 pages, 2018.

[14] Z. Biolek, D. Biolek, and V. Biolkova, "(Co)content in circuits with memristive elements," IEEE Transactions on Circuits and Systems I: Regular Papers, vol. 62, no. 2, pp. 488-496, 2015.

[15] E. Noether, "Invariante variationsprobleme," in Nachrichten von der Gesellschaft der Wissenschaften zu Göttingen, Mathematisch-Physikalische Klasse, pp. 235-257, Göttinger Akademie der Wissenschaften, Göttingen, Germany, 1918.

\section{Conflicts of Interest}

The authors declare that there are no conflicts of interest regarding the publication of this paper. 


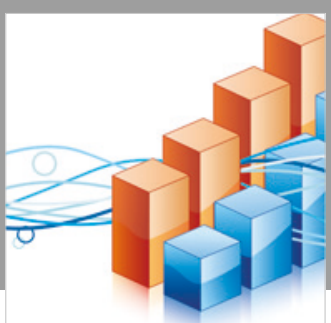

Advances in

Operations Research

\section{-n-m}
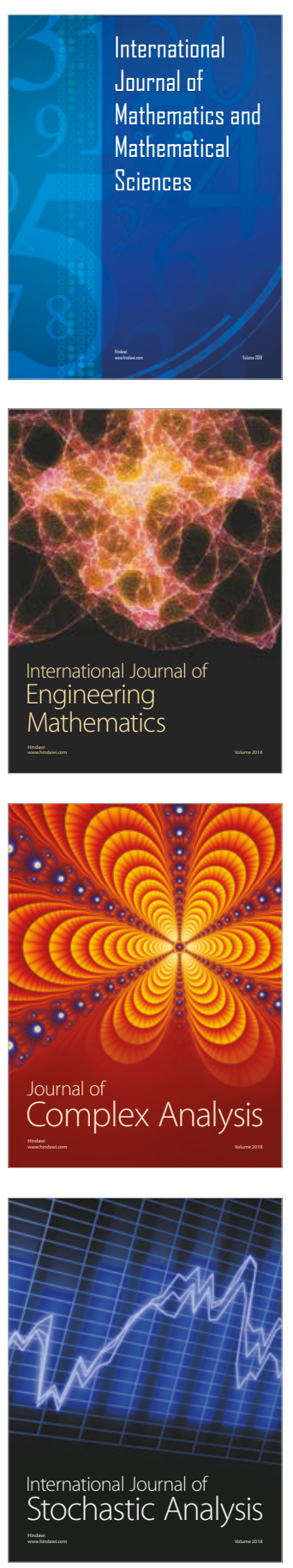
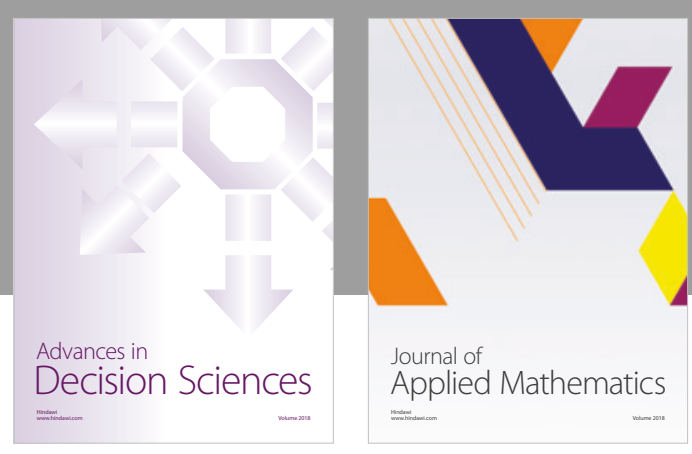

Journal of

Applied Mathematics
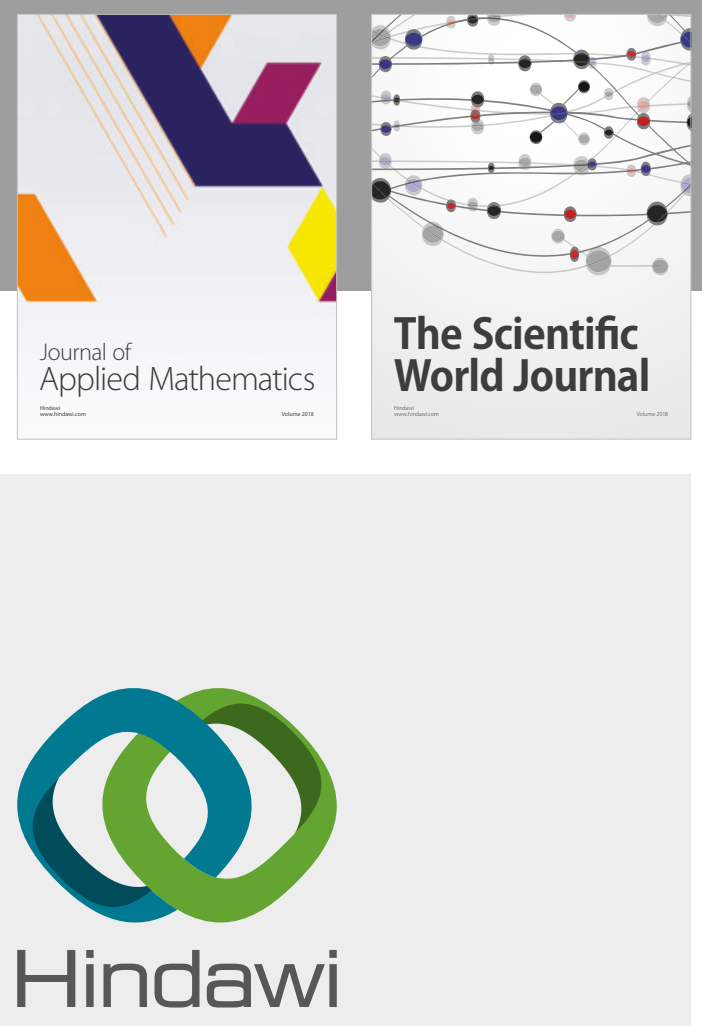

Submit your manuscripts at

www.hindawi.com

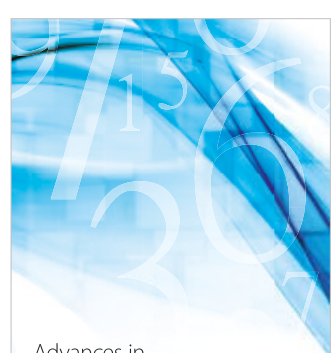

Advances in
Numerical Analysis
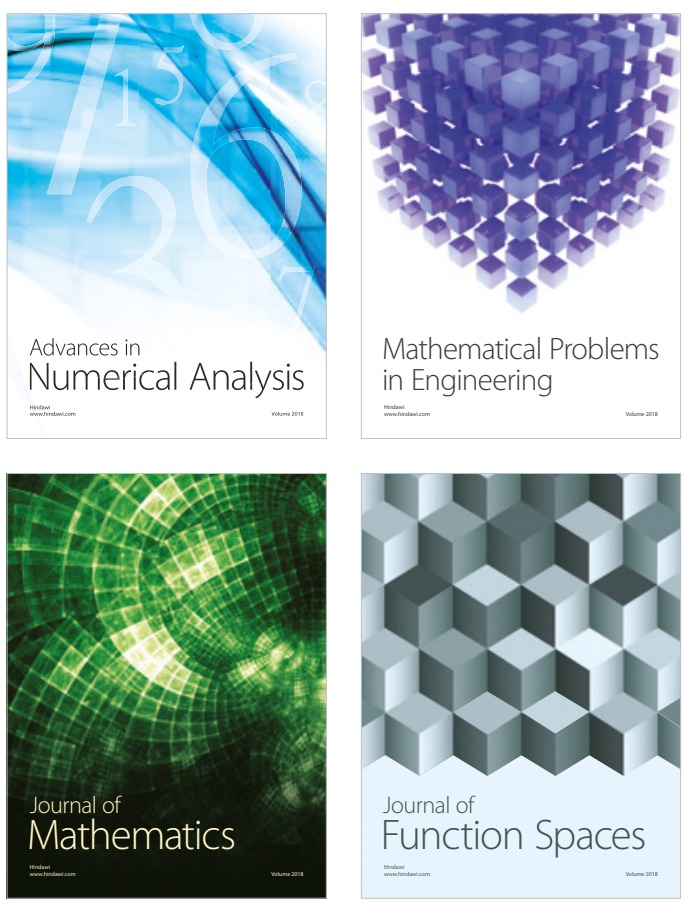

Mathematical Problems in Engineering

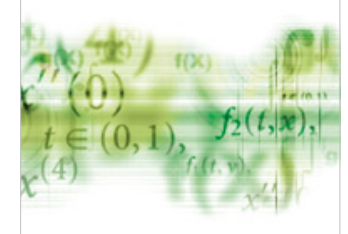

International Journal of

Differential Equations

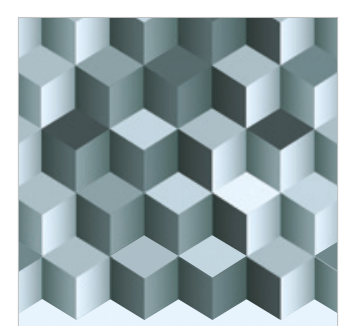

Journal of

Function Spaces
The Scientific

World Journal

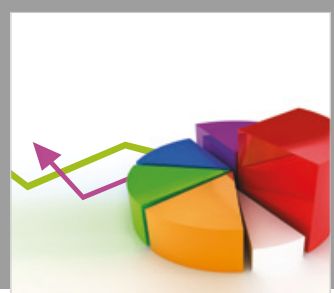

Journal of

Probability and Statistics
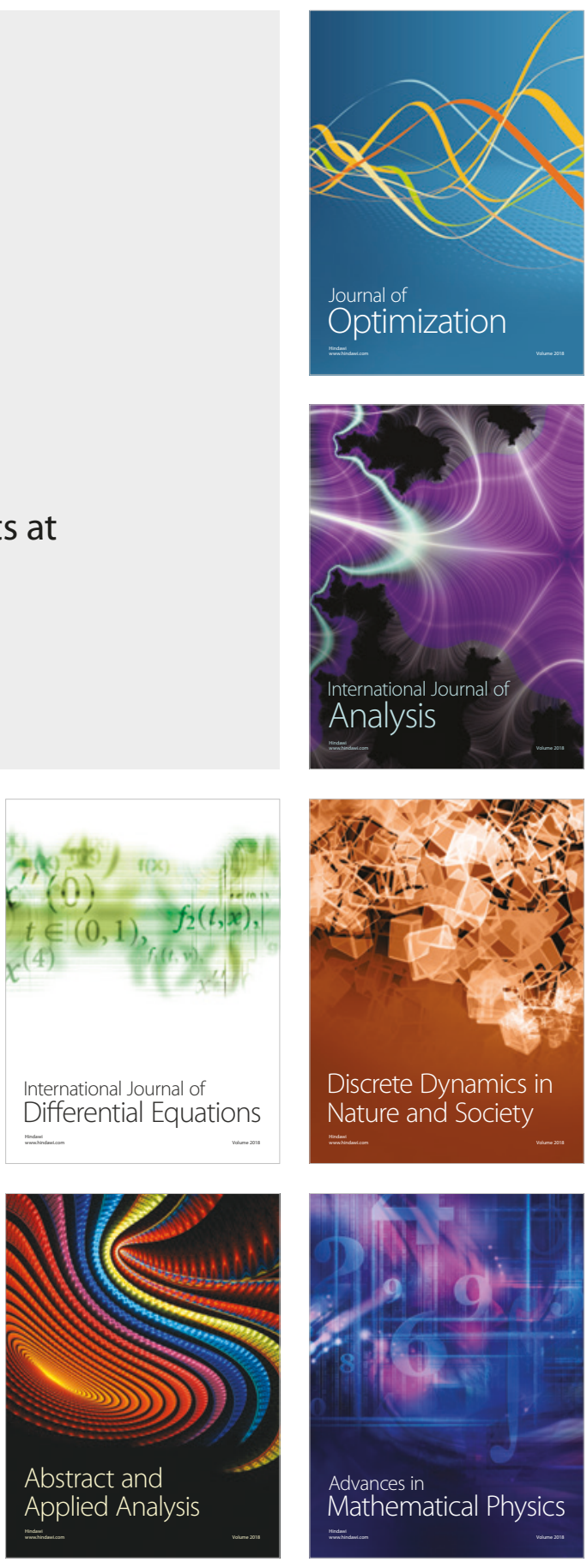